\title{
CNC Setting and \\ Operation Workbook
}




\section{City and Guilds Co-publishing Series}

City and Guilds of London Institute has a long history of providing assessments and certification to those who have undertaken education and training in a wide variety of technical subjects or occupational areas. Its business is essentially to provide an assurance that pre-determined standards have been met. That activity has grown in importance over the past few years as government and national bodies strive to create the right conditions for the steady growth of a skilled and flexible workforce.

Both teachers and learners need materials to support them as they work towards the attainment of qualifications, and City and Guilds is pleased to be working with several distinguished publishers towards meeting that need. It has been closely involved in the planning, author selection and text appraisal, although the opinions expressed in the publications are those of the individual authors and are not necessarily those of the Institute.

City and Guilds is fully committed to the projects listed below and is pleased to commend them to teaching staff, students and their advisers.

Carolyn Andrew and others, Business Administration Level I and Business Administration Level II, John Murray

Chris Cook, Assessor Workbook, Macmillan

David Minton, Teaching Skills in Further and Adult Education, Macmillan

Graham Morris and Lesley Reveler, Retail Certificate Workbook (Levels 1 and 2), Macmillan

Peter Riley(consultant editor), Computer-aided Engineering, and associated Workbooks: CNC Setting and Operation; CNC Part Programming; Computer-aided Draughting; Robot Technology; Programmable Logic Control, Macmillan

Barbara Wilson, Information Technology: the Basics, Macmillan Caroline Wilkinson, Information Technology in the Office, Macmillan 


\section{CNC Setting and Operation Workbook}

\section{Tom Renshaw}

Department of Engineering Technology

Blackpool and The Fylde College

Consultant Editor: Peter Riley

Formerly Head of Department of Engineering

Technology, Blackpool and The Fylde College 
All rights reserved. No reproduction, copy or transmission of this publication may be made without written permission.

No paragraph of this publication may be reproduced, copied or transmitted save with the written permission or in accordance with the provisions of the Copyright, Designs and Patents Act 1988, or under the terms of any licence permitting limited copying issued by the Copyright Licensing Agency, 90 Tottenham Court Road, London WiP 9HE.

Any person who does any unauthorised act in relation to this publication may be liable to criminal prosecution and civil claims for damages.

First published 1994 by

THE MACMILLAN PRESS I.TD

Houndmills, Basingstoke, Hampshire RG21 2XS

and London

Companies and representatives

throughout the world

ISBN 978-1-349-12687-3

DOI 10.1007/978-1-349-12685-9

A catalogue record for this book is available from the British Library

Typeset by EXPO Holdings, Malaysia

\section{Acknowledgements}

Thanks are due to the following for permission to use photographs: Cincinnati Milacron (page 3), Norelen (page 16). 


\section{Contents}

$\begin{array}{lr}\text { Introduction } & 1\end{array}$

How to use this book 2

Safety note $\quad 3$

Learning Assignment 1 Position machine axes 4

Task 1.1 Absolute and incremental positioning 5

$\begin{array}{lll}\text { Task 1.2 Checking table limits of travel } & 7\end{array}$

Task 1.3 Manual positioning of tool in all axes 7

Learning Assignment 2 Methods of guarding 8

$\begin{array}{ll}\text { Protecting the operator } & 8\end{array}$

Protecting the machine and/or workpiece $\quad 8$

Task 2.1 Methods of protection on CNC machines 9

Task 2.2 Operating the machine $\mathrm{Z}$ axis lock 9

Task 2.3 Spindle motor protection on a turning centre 9

$\begin{array}{lll}\text { Task 2.4 Checking interlock guards } & 10\end{array}$

Learning Assignment 3 Machine datums 11

$\begin{array}{lll}\text { Task 3.1 Setting machine to datum position } & 12\end{array}$

$\begin{array}{lll}\text { Task } 3.2 & \text { Re-setting machine override } & 12\end{array}$

$\begin{array}{lll}\text { Task 3.3 Transferring machine datum to workpiece datum } & 13\end{array}$

Task 3.4 Component location using work co-ordinates 13

Learning Assignment 4 Work-holding 14

$\begin{array}{ll}\text { Selecting clamps } & 14\end{array}$

$\begin{array}{lll}\text { Task 4.1 Component setting } & 15\end{array}$

$\begin{array}{ll}\text { Task 4.2 Component clamping } & 15\end{array}$

$\begin{array}{lll}\text { Learning Assignment 5 NC tooling } & 17\end{array}$

$\begin{array}{lll}\text { Task 5.1 Working practices } & 17\end{array}$

$\begin{array}{lll}\text { Task } 5.2 \text { Tool tip selection } & 18\end{array}$

$\begin{array}{lll}\text { Task } 5.3 \text { Tool identification } & 18\end{array}$

Learning Assignment 6 Tool changing 20

$\begin{array}{ll}\text { Manual tool changing } & 20\end{array}$

Automatic tool changing using magazines or carousels 20

$\begin{array}{ll}\text { Automatic tool changing using turrets } & 20\end{array}$

$\begin{array}{lll}\text { Task 6.1 Tool selection: turning } & 21\end{array}$

$\begin{array}{lll}\text { Task } 6.2 \text { Tool selection: milling } & 21\end{array}$

Learning Assignment 7 Tool offset facilities: turning 23

$\begin{array}{ll}\text { Programming information } & 23\end{array}$

Task 7.1 Setting a tool offset in the X axis 23

Learning Assignment 8 Tool length offsets: milling 25

$\begin{array}{ll}\text { Methods of tool offset measurement } & 25\end{array}$

Task 8.1 Setting TLO values manually on a machine 26

Task 8.2 Set TLO values away from the machine 26 
Learning Assignment 9 Tool setting 27

$\begin{array}{ll}\text { Pre-setting } & 28\end{array}$

Setting on the machine (turning) 28

Qualified tooling $\quad 28$

Task 9.1 Pre-setting tools using a fixture 29

Task 9.2 Pre-setting tools by measurement on machine 29

$\begin{array}{ll}\text { Tool nose radius compensation (TNRC) } & 30\end{array}$

Learning Assignment 10 NC program listing and analysis 31

$\begin{array}{lll}\text { Task 10.1 Program analysis } & 32\end{array}$

Task 10.2 Program efficiency $\quad 32$

Learning Assignment 11 Input, edit and store data 34

$\begin{array}{lll}\text { Task } 11.1 & \text { Data storage } & 34\end{array}$

Task 11.2 Data transfer $\quad 35$

Learning Assignment 12 Program proving 36

$\begin{array}{ll}\text { Testing method 1: Dry run } & 37\end{array}$

Testing method 2: Step through block by block 37

$\begin{array}{ll}\text { Testing method 3: Graphic simulation } & 37\end{array}$

$\begin{array}{lll}\text { Task 12.1 Program proving } & 38\end{array}$

$\begin{array}{lll}\text { Task 12.2 Program proving and correction } & 38\end{array}$

Learning Assignment 13 Tape handling 40

$\begin{array}{lll}\text { Task 13.1 Tape editing } & 41\end{array}$

Task 13.2 Tape loading and re-winding $\quad 42$

Learning Assignment 14 Tape preparation 43

$\begin{array}{ll}\text { Code standards for punched tape } & 43\end{array}$

Preparing the paper tape $\quad 45$

Task 14.1 Tape preparation $\quad 45$

$\begin{array}{lr}\text { Glossary } & 47\end{array}$

$\begin{array}{lr}\text { Index } & 49\end{array}$ 